\title{
LA INVENCIÓN DE PROBLEMAS COMO TAREA ESCOLAR
}

\section{Ma Fernanda Ayllón Blanco e Isabel A. Gómez Pérez}

\section{RESUMEN}

El trabajo presentado expone los beneficios que la tarea de inventar problemas aporta a la construcción del conocimiento matemático y a su éxito educativo. Se señalan como factores positivos de dicha tarea el aumento del conocimiento matemático, la motivación y la creatividad, la disminución de la ansiedad hacia las matemáticas, la superación de errores matemáticos y la posibilidad de que el profesorado la utilice como instrumento evaluador. Se justifica la pertinencia de indagar en el campo de la invención de problemas a partir de una doble vía: las investigaciones realizadas en Educación Matemática y las directrices que marcan diferentes documentos curriculares. Este artículo presenta algunos de los estudios más relevantes que se han realizado hasta el momento donde se relaciona la invención de problemas por escolares con campos como la resolución de problemas, la instrucción, la tecnología, la evaluación y los métodos de enseñanza.

Palabras clave: problemas, invención de problemas, invención de problemas por escolares, Educación Matemática.

\section{TITLE: PROBLEM POSING AS A SCHOOL TASK}

\section{ABSTRACT}

This essay deals with the benefits that the task of problem posing brings to the construction of mathematical knowledge and to its educational success. Positive factors of such a task are: the increase of mathematical knowledge, motivation and creativity; the decrease of anxiety, the overcoming of mathematical mistakes and as a possible instrument of evaluation for the teacher. We justify the relevance of studying the field of problem posing by starting from a double channel: the research carried out in Mathematical Education and the guidelines that different curricular documents lay out. This article presents some of the most relevant studies that have been carried out up to the present where problem posing by pupils is related to fields like problem solving, instruction, technology, evaluation and teaching methods.

Keywords: problems, problem posing, problem posing by school children, Mathematical Education.

Correspondencia con las autoras: Mả Fernanda Ayllón Blanco. < mayllonblanco@eulainmaculada.com > Isabel A. Gómez Pérez. < isabelgomez@eulainmaculada.com>. Centro de Magisterio La Inmaculada (Adscrito a la Universidad de Granada). Original recibido: 08-II-13. Original aceptado: 21-02-14 


\section{Invención de problemas}

La investigación en educación matemática considera que el trabajo con problemas es imprescindible para que el individuo construya de manera significativa el Conocimiento Matemático (Freudenthal, 1973, Kilpatrick, 1987 y Polya, 1965); asimismo advierte que cuando un sujeto inventa un problema está alcanzando niveles elevados de razonamiento que hacen posible la construcción del conocimiento matemático. Se justifica la necesidad de indagar en el campo de la invención de problemas a partir de una doble vía. Una primera vía parte de las investigaciones aportadas en Educación Matemática donde se fijan como objetivos prioritarios de la enseñanza-aprendizaje de las matemáticas el planteamiento y la resolución de problemas (Noda, 2000). La segunda vía de justificación se halla en diferentes documentos curriculares. En la etapa de Educación Primaria, los correspondientes al Real Decreto I5 I3/2006 del MEC (Ministerio de Educación y Ciencia) y el Decreto 230/2007 de la Junta de Andalucía, y a distinto nivel curricular en National Council of Teachers of Mathematics (NCTM 1980, 199I y 2000).

La acción de inventar problemas es una actividad intelectual y una manera eficiente de aprender matemáticas (Freudenthal, 1973, Kilpatrick, 1987 y Polya, 1965). Cuando un individuo inventa un problema alcanza niveles de reflexión complejos, por tanto llega a una etapa de razonamiento donde es posible construir el conocimiento matemático.

Christou, Mousoulides, Pittalis y Pitta-Pantazi (2005) estudian el pensamiento que necesita un alumno para inventar problemas. En su investigación establecen que en esta tarea se ven envueltos distintos procesos cognitivos los cuales se identifican como: editar información, seleccionar información, comprender y organizar información y traducir la información de una forma de representación a otra. Reconocen que estos procesos son imprescindibles en la invención de problemas y encuentran que la formulación de problemas aporta grandes beneficios a la enseñanza-aprendizaje de las matemáticas, contribuyendo a que el alumno pueda alcanzar un aprendizaje exitoso en este ámbito ya que habrá establecido relación entre los distintos conceptos matemáticos. Abu-Elwan (1999) plantea la necesidad de potenciar este trabajo en el aula, por lo que se recomienda que los profesores de matemáticas provean abundantes y variadas oportunidades a sus alumnos, tanto para aprender a resolver problemas como a inventar problemas en una gran cantidad de situaciones. 


\section{Aspectos positivos de la invención de problemas en la educación matemática}

Los estudios realizados en Educación Matemática señalan diferentes factores positivos que la invención de problemas aporta para la enseñanza-aprendizaje de las matemáticas. Uno de estos factores positivos se refiere al aumento del conocimiento matemático, debido a que esta tarea obliga a crear conexiones entre distintos conocimientos que se poseen separadamente. El inventor de problemas leerá, examinará datos y pensará críticamente (Davidson y Pearce, 1988), discutirá ideas, estrategias y soluciones a la vez que las cuestionará (Whitin, 2006). Con frecuencia habrá de generalizar, siendo necesario escribir con claridad, exactitud y organización (Burçin, 2005).

Otro factor positivo apunta a la motivación. La comunidad matemática considera que una buena motivación llevará a acrecentar el rendimiento de los estudiantes, ayudando a que alcancen el tan ansiado éxito educativo. Para ello, Akay y Boz (2010) y Silver (1994) proponen como instrumento de motivación la invención de problemas. Se apoyan en que esta tarea fomenta una actitud positiva en clase de matemáticas, debido a que el trabajo con problemas despierta en los estudiantes la curiosidad y la motivación.

Un tercer factor positivo que aporta la invención de problemas está relacionado con la ansiedad que, en ciertos estudiantes, produce su relación con las matemáticas. Se considera que al inventar problemas se fomenta una disposición más favorable y responsable hacia las matemáticas ayudando a rebajar la ansiedad de los estudiantes hacia las mismas. Burçin (2005) y Song, Yim, Shin y Lee (2007) afirman que la invención de problemas disminuye el miedo y la preocupación que algunos estudiantes sienten por las matemáticas.

Un cuarto aspecto positivo alude a la superación de errores matemáticos que frecuentemente cometen los estudiantes. Brown y Walter (1993) afirman que la invención de problemas estimula al alumno a elegir la información que ha de utilizar en la resolución del problema y a seleccionar con qué datos desea operar, haciendo que se produzca una disminución de errores resolutivos.

La creatividad es el quinto elemento positivo. Se determina que la invención de problemas aporta grandes beneficios al desarrollo de la creatividad de los estudiantes. Ellerton (1986) y Krutetskii (1969) evidencian una relación entre el grado de creatividad y competencia matemática y la habilidad para inventar problemas. 
Silver (1994) estudia la creatividad de los estudiantes atendiendo a tres variables: fluidez asociándola al número de problemas generados, flexibilidad la que relaciona con el número de categorías diferentes de los problemas propuestos y el grado de originalidad que lo vincula con el número de soluciones que admiten los problemas propuestos. Esta investigación le lleva a afirmar que existe una relación directa entre el nivel de creatividad de los estudiantes y la destreza que presentan cuando estos inventan problemas.

Un sexto factor positivo se relaciona a la tarea evaluadora del profesorado. A través de la de invención de problemas, el profesor podrá conocer las habilidades que tienen sus alumnos para usar su conocimiento matemático (Ayllón, 2005; Lin, 2004; Sheikhzade, 2008), asimismo permitirá analizar los procesos de pensamiento matemático de los sujetos evaluados. Por tanto, la invención de problemas permite evaluar, en los estudiantes, su conocimiento, su manera de razonar y su desarrollo conceptual.

\section{Investigaciones en invención de problemas matemáticos}

La bibliografía especializada sostiene que la invención de problemas ha recibido poca atención explícita en las investigaciones, si bien es reconocida como uno de los campos de investigación sobre problemas. Cruz (2006) estudió la producción en investigación sobre invención de problemas. Este estudio le llevó a afirmar que, a pesar de su importancia, la invención de problemas no ha sido globalmente tratada como parte del currículo de matemáticas y tampoco han sido suficientemente sistemáticas las investigaciones relacionadas en este tema (Kilpatrick, 1987).

Se encuentran algunas investigaciones sobre formulación de problemas en la literatura pero muchos de estos estudios están relacionados con la reformulación de problemas ya enunciados. Numerosos estudios presentan un vínculo estrecho entre la invención y la resolución de problemas (Kilpatrick, 1987; Silver, 1994) y muestran que una herramienta importante en la instrucción sobre resolución de problemas es la invención de problemas que proporciona mayor comprensión a este desempeño y contribuye a aumentar en los alumnos el conocimiento matemático. La práctica que poseen los alumnos en materia de resolución de problemas constituye un elemento que influye fuertemente en la relación establecida entre la invención y la resolución de problemas.

Se distinguen dos líneas de estudio en las investigaciones realizadas sobre invención de problemas. La primera corresponde a la invención de problemas por 
parte de escolares y la segunda a la invención de problemas por profesores en ejercicio y formación. Aunque esta segunda línea no la vamos a considerar en este artículo es conveniente advertir que coincide con la primera en que es necesario incluir la invención de problemas en las tareas propias de estudiantes y profesores, al considerarla como uno de los elementos involucrados para que el estudiante sea competente matemáticamente.

\section{Invención de problemas por escolares}

Seguidamente se recogen algunas de las investigaciones consultadas sobre invención de problemas vinculadas a diferentes campos donde los participantes son escolares.

\section{I. Invención y resolución de problemas}

En ocasiones se presenta la invención de problemas como una variable que ayuda a predecir la capacidad de un individuo para resolver problemas. Kilpatrick (1987) manifiesta que los estudiantes que son capaces de inventar problemas matemáticos son buenos resolutores de problemas.

Silver ( 1994) evidencia que la invención de problemas influye de manera positiva en la capacidad que tienen los estudiantes para resolver problemas. Enumera diferentes razones con las que justifica esta afirmación asegurando que el beneficio de dicha tarea no es únicamente para el alumnado sino también para el profesorado. Entre ellas destacamos: que la invención de problemas está relacionada con la creatividad del individuo; que es un instrumento que favorece que los estudiantes sean mejores resolutores de problemas; que ayuda a que los estudiantes comprendan mejor los conceptos matemáticos; que mejora la aptitud y disposición que los estudiantes tienen hacia la materia y que permite el aprendizaje autónomo en el alumno.

Silver y Cai (1996) realizan un estudio donde analizan la relación entre la capacidad de proponer problemas y la de resolver problemas. Entre sus resultados obtuvieron que los estudiantes frente a una situación conocida, inventaron numerosos problemas que se podían resolver, donde sus enunciados incluso tenían un nivel semántico y sintáctico elevado. También observaron que los alumnos considerados buenos resolutores de problemas formularon más cantidad de problemas y más complejos que los sujetos considerados malos resolutores. Los autores aseguran 
que existe una fuerte correlación entre la resolución de problemas y la invención de problemas, y concluyen su estudio advirtiendo que los alumnos considerados buenos resolutores de problemas, inventan más problemas y más complejos, que los sujetos considerados malos resolutores.

Carlson y Bloom (2005) y Cifarelli y Cai (2005) dicen que cuando los alumnos resuelven un problema cuestionan sus acciones y objetivos y que esta situación les lleva a la formulación de un nuevo problema. Por tanto advierten que la invención de problemas ayuda al estudiante a progresar en el proceso de resolución de problemas. Sin embargo, en un estudio posterior, Penalva, Posadas y Roig (2010) afirman que no está clara la relación entre la resolución y el planteamiento de problemas. Su investigación concluye en que no se encuentra un vínculo entre los buenos resolutores de problemas y los inventores de problemas y que existe una relación compleja entre la manera de resolver y formular problemas. Estos resultados se parecen a los expuestos por Crespo (2003) quien afirma que un buen resolutor de problemas no tiene por qué ser un buen inventor de problemas pero contradicen los obtenidos por Cai (1998), Leung (1996), Silver (1994) y Silver y Cai (en 1996).

\subsection{Invención de problemas y desarrollo de habilidades}

Burçin (2005) analiza si la invención de problemas fomenta en los alumnos una actitud positiva hacia la probabilidad y una mejor comprensión de la misma. En su estudio participaron dos grupos de estudiantes. En el primero de ellos se siguió la instrucción tradicional para la enseñanza de la probabilidad y en el segundo se instruyó en invención de problemas. El resultado fue que el segundo grupo obtuvo mejores resultados que el primero, en el que se había utilizado una metodología tradicional.

En 2007, Nicolaou y Pilippou buscaron relaciones entre sus creencias sobre la eficacia que tenían 87 alumnos de quinto y 87 de sexto grado en inventar problemas, su habilidad inventando problemas y su competencia matemática. Observaron que existe una alta correlación entre la eficacia en inventar problemas con el logro en matemáticas de estos estudiantes.

El trabajo aportado por Alias, Ghazali y Ayub (2009), donde participan estudiantes de 8-9 años de edad de tres clases con competencia matemática diversa, en que se presentaron sentencias numéricas de sumas y restas de uno, dos y tres dígitos sobre las cuales debían inventar un problema que pudieran resolver con cada una de dichas sentencias mostró que: I) conforme avanza el curso escolar los niños 
inventan problemas más complejos, 2) los estudiantes se hallan cómodos en la realización de esta tarea, 3) casi todos los estudiantes inventan problemas a partir de sus experiencias personales y 4) al inventar problemas estos estudiantes utilizan estrategias que contribuyen a que sean más competentes en la resolución de problemas.

\subsection{Invención a través de la tecnología}

Christou, Mousoulides, Pittalis y Pitta-Pantazi (2005) estudian la utilidad de herramientas tecnológicas en la invención y resolución de problemas de geometría. Obtuvieron entre otros resultados que el software de geometría dinámica es un instrumento útil en la resolución e invención de problemas provocando que el alumno realice experimentos, conjeturas y generalizaciones.

Birch y Beal (2008) trabajaron la invención de problemas a partir del sistema tutorial "Animal Watch". Observaron que los estudiantes, al utilizar la invención de problemas en su aprendizaje, aprendieron sobre animales. Los resultados garantizan que la motivación del alumno se incrementa cuando plantea problemas a partir de situaciones reales y facilita el aprendizaje, tanto de contenidos matemáticos como de otras disciplinas.

\subsection{Invención como evaluación}

Mestre (2002) utiliza la invención de problemas para el estudio de procesos cognitivos. A través de esta tarea analiza el razonamiento, el desarrollo conceptual y la transferencia de conceptos en diferentes contextos para identificar el conocimiento de los estudiantes. Concluye que la invención de problemas posee una alta capacidad de transferencia de conocimientos y constituye un instrumento evaluador de la comprensión de los conceptos físicos que tienen los alumnos.

English (2003) manifiesta que la invención de problemas permite indagar sobre la comprensión de los estudiantes de los conceptos matemáticos y sus relaciones.

Alexander y Ambrose (2010) animan a los profesores a utilizar la invención de problemas como herramienta de evaluación. Las investigadoras aseguran que la invención de problemas les permitió identificar el nivel de comprensión que tenían los alumnos sobre el concepto de fracción y sus dificultades, y afirman que la tarea propuesta permite contextualizar significativamente las fracciones. 


\subsection{Invención como método de enseñanza}

Santos (200l y 2008) presenta la invención de problemas como una metodología para la enseñanza y aprendizaje de las matemáticas. Manifiesta que si este proceso es adecuado, hará que los estudiantes produzcan problemas bien formulados que, en el aula de matemáticas, llevará a que los alumnos adquieran un correcto aprendizaje. Considera como factores esenciales de este proceso: la acción, el diálogo, un enfoque de las matemáticas desde fuera y la aceptación del vínculo entre sociedad y escuela.

\section{Conclusión y recomendación}

A pesar de que los investigadores afirman que la invención de problemas conlleva importantes beneficios para el aprendizaje matemático y el desarrollo intelectual del individuo, las investigaciones en este campo aún son insuficientes.

Queda evidenciado que la invención de problemas contribuye a la construcción del conocimiento lógico-matemático e incrementa de forma considerable el desarrollo del razonamiento en el estudiante. El estudiante, al inventar problemas, considera la matemática como algo propio, crea situaciones más cercanas y reales a él, haciendo que su implicación en la tarea matemática sea total. Esto hace que se incrementen las ganas de aprender en muchos alumnos y disminuya el temor que en algunos de ellos genera el aprendizaje matemático.

Todo profesor anhela que sus alumnos adquieran conocimiento matemático, siendo uno de sus objetivos principales desarrollar su razonamiento. El trabajo con problemas es clave para alcanzar tal propósito, pues junto con los conceptos numéricos constituye el eje vertebrador de casi todos los conocimientos matemáticos. Se ha visto que la invención de problemas aparece fuertemente vinculada a la resolución de problemas. Por lo general, el estudiante que inventa problemas sabe cómo resolverlos, así como el que es un buen resolutor de problemas es capaz de inventar problemas. Para el profesorado, es muy interesante el papel evaluador que tiene la invención de problemas. Esta tarea le permitirá indagar en cómo piensan matemáticamente sus alumnos, el grado de comprensión que poseen de determinados conceptos matemáticos, las razones por las que comenten ciertos errores y las estrategias que utilizan.

El profesor también puede utilizar la invención de problemas como método de enseñanza, como contenido curricular y como vínculo al uso de las nuevas tecnologías. Por lo que esta tarea no se presenta de forma aislada en la construcción del conocimiento matemático. 
Por todo esto se recomienda a los profesores que incluyan la invención de problemas en las tareas que proponen a sus escolares. Es necesario que se forme en dicha tarea tanto a los profesores que están en activo como a los futuros profesores, desarrollando en ellos esta habilidad. Esto les ayudará a motivar a su alumnado, para que se enfrente sin miedos a problemas que han de resolver o que han de inventar y hacer que el alumno confíe más en sus capacidades y así sean competentes matemáticamente.

\section{Referencias}

Abu-Elwan, R. (1999). The development of mathematical problem posing skills for prospective middle school teachers. En A. Rogerson (Ed.), Proceedings of the International Conference of the $21^{\text {st }}$ Century Project: Societal Challenges, Issues and Approaches (Vol. 2, pp. I-8). El Cairo, Egipto: the $2 \mathrm{I}^{\text {st }}$ Century Project. Recuperado el 08/07/2010 de http://dipmat.math.unipa.it/ grim/EAbu-elwan8.PDF

Akay, H. y Boz, N. (20 I0). The effect of problem posing oriented analyses-II course on the attitudes toward mathematics and mathematics self-efficacy of elementary prospective mathematics teachers. Australian Journal of Teacher Education, 35 (I), $59-65$.

Alexander, C. y Ambrose, R. C. (2010). Digesting student-authored story problems. Mathematics Teaching in the Middle School, 16 (I), 27-33.

Alias, R., Ghazali, M. y Ayub, A. (2009, Noviembre). Student's problem posing strategies: implications to student's mathematical problem solving. Presentado en la $5^{\text {th }}$ Asian Mathematical Conference, Kuala Lumpur, Malasia.

Ayllón, M. F. (2005). Invención de problemas con números naturales, enteros negativos y racionales. Tarea para profesores de educación primaria en formación. Trabajo de investigación tutelada, Universidad de Granada, España.

Birch, M. y Beal, C. R. (2008). Problem posing in AnimalWatch: An interactive system for student-authored content. Presentado en el $21^{\text {st }}$ International the Florida Artificial Intelligence Research Society (FLAIRS) conference, Coconut Grove, Florida. Recuperado el I4/0I/2009 de http://learn.cs.arizona.edu/pages/ viewpageattachments.action?pageld $=1016823$.

Brown, S. I. y Walter, M. I. (1993). Problem posing. Hillsdale, NJ: Lawrence Erlbaum Associates. 
Burçin, B. (2005). The effect of instruction with problem posing on tenth grade students' probability achievement and attitudes toward probability. Tesis doctoral, Universidad de Ankara, Turquía.

Cai, J. (1998). An investigation of U.S. and Chinese students' mathematical problem posing and problem solving. Mathematics Education Research Journal, 10 (I), 37-50.

Carlson, M. y Bloom, I. (2005). The cyclic nature of problem solving: An emergent multidimensional problem-solving framework. Educational Studies in Mathematics, 58 (I), 45-75.

Christou, C., Mousoulides, N., Pittalis, M. y Pitta-Pantazi, D. (2005). Problem solving and problem posing in a dynamic geometry environment. The Montana Mathematics Enthusiast, 2 (2), I25-I43.

Cifarelli, V. y Cai, J. (2005). The evolution of mathematical explorations in open ended problem solving situations. Journal of Mathematical Behavior, 24 (3-4), 302 324.

Consejería de Educación y Ciencia (2007). Decreto 230/2007, de 30 de agosto, por el que se desarrolla el currículum correspondiente a educación primaria. BOJA, 17I, 4-23.

Crespo, S. (2003). Learning to pose mathematical problems: exploring changes in preservice teachers' practices. Educational Studies in Mathematics, 52 (3), 243-270.

Cruz, M. (2006). A mathematical problem-formulating strategy. International Journal for Mathematics Teaching and Learning, 7 de Diciembre. Recuperado el 24/04/2009 de http://www.cimt.plymouth.ac.uk/journal/ramirez.pdf

Davidson, D. y Pearce, D (1988). Using writing activities to reinforce mathematics instruction. Arithmetic Teacher, 35 (I8), 42-45.

Ellertoh, N. F. (1986). Children's made up mathematics problems. A new perspective on talented mathematicians. Educational Studies in Mathematics, 17 (3), 26I-27I.

English, L. (2003). Engaging students in problem posing in an inquiry-oriented mathematics classroom. En F. Lester y C. Randall (Eds.), Teaching mathematics through problem solving (pp. 187-198). Reston, VA.: National Council of Teachers of Mathematics. 
Freudenthal, H. (1973). Mathematics as an educational task. Dordrecht, Noruega: Reidel.

Freudenthal, H. (199I). Revisiting mathematics education. Dordrecht, Noruega: Kluwer Academic Publishers.

Kilpatrick, J. ( 1987). Problem formulating: where do good problems come from? En A. H. Schoenfeld (Ed.), Cognitive science and mathematics education (pp. I23127). Hillsdale, NJ: Laurence Erlbaum Associate.

Krutetskii, V. A. (1969). An investigation of mathematical abilities in schoolchildren. En J. Kilpatricky e I. Wirszup (Eds.), Soviet studies in the psychology of learning and teaching mathematics (Vol. 2, pp. 5-57). Chicago, IL: University of Chicago Press.

Leung, S. (1996). Problem posing as assessment: Reflections and reconstructions. The Mathematics Educator, I (2), I59-I7I.

Lin, P. J. (2004). Supporting teachers on designing problem-posing tasks as a tool of assessment to understand students' mathematical learning. En M. Hoines y A. Fuglestad (Eds.), Proceedings of the $28^{\text {th }}$ annual meeting of the International Group for the Psychology of Mathematics Education (Vol. 3, pp. 257-264). Bergen, Noruega: Bergen University College.

Mestre J. P. (2002). Probing adults' conceptual understanding and transfer of learning via problem posing. Journal of Applied Developmental Psychology, 23 (I), 9-50.

Ministerio de Educación y Ciencia (MEC) (2006). Real Decreto 1513/2006, de 7 de diciembre, por el que se establecen las enseñanzas mínimas de la Educación primaria. BOE, 293, 43053-43102.

National Council of Teachers of Mathematics (1980). Problem solving in school mathematics. 1980 Yearbook. Reston, VA: Autor.

National Council of Teachers of Mathematics (1991). Estándares curriculares y de evaluación para la educación matemática. Sociedad Andaluza de Educación Matemática THALES: Granada

National Council of Teachers of Mathematics (2000). Principles and standards for school mathematics. Reston, VA: Autor.

Nicolaou, A. y Pilippou, G. (2007). Eficacy belief, problem posing, and mathematics achievement. En D. Pitta-Pantazi, y G. Philippou (Eds.), Proceedings of the V Congress 
of the European Society for Research in Mathematics Education (pp. 308-3 I 7). Larnaca, Chipre: Department of Education, University of Cyprus.

Noda, A. (2000). Aspectos epistemológicos y cognitivos de la resolución de problemas de matemáticas, bien y mal definidos. Un estudio con alumnos del primer ciclo de la ESO y maestros en formación. Tesis doctoral, Universidad de la Laguna, España.

Penalva, M. C., Posadas, J. A. y Roig, A. I. (20 I0). Resolución y planteamiento de problemas: contextos para el aprendizaje de la probabilidad. Educación Matemática, 2 (3), 23-54. Trillas.

Polya, G. (1965). Cómo plantear y resolver problemas. México DC, México:

Santos, M. C. (200I). Problem posing and problematization in learning and teaching mathematics. Adult Education and Development, 57, I07-I 21 .

Santos, L. M. (2008). La resolución de problemas matemáticos. Avances y perspectivas en la construcción de una agenda de Investigación y práctica. En $R$. Luengo, B. Gómez, M. Camacho, y L. Blanco (Eds.). Investigación en Educación Matemática XII (pp. I59-192). Badajoz: Sociedad Española de Investigación en Educación Matemática.

Sheikhzade, M. (2008). Promoting skills of problem-posing and problem-solving in making a creative social studies classroom. Presentado en la 4th Global Conference, Oxford. Recuperado el 9/10/2010 de http://www.inter-disciplinary.net/ati/ education/cp/ce4/Sheikhzade\%20paper.pdf.

Silver, E. A. (1994). On mathematical problem posing. For the Learning of Mathematics, I4 (I) 19-28.

Silver, E. A. y Cai, J. (1996): An analysis of arithmetic problem posing by middle school students. Journal for Research in Mathematics Education, 27 (5), 52 I -539.

Song, S., Yim, J., Shin, E. y Lee, H. (2007). Posing problems with use the 'what if not?' strategy in NIM game. En J. H. Woo, H. C. Lee, K. S. Park, y D. Y. Seo, (Eds.). Proceedings of the $3 \mathrm{I}^{\text {st }}$ Conference of the International Group for the Psychology of Mathematics Education (Vol. 4, pp. 193-200). Seúl, Corea del Sur: PME.

Whitin, D. J. (2006). Problem posing in the elementary classroom. Teaching Children Mathematics, I3 (I), I4-I8. 